\title{
QUASI-DEFINITENESS OF GENERALIZED UVAROV TRANSFORMS OF MOMENT FUNCTIONALS
}

\author{
D. H. KIM AND K. H. KWON
}

Received 11 March 2001

When $\sigma$ is a quasi-definite moment functional with the monic orthogonal polynomial system $\left\{\mathrm{P}_{\mathfrak{n}}(\mathrm{x})\right\}_{\mathfrak{n}=0}^{\infty}$, we consider a point masses perturbation $\tau$ of $\sigma$ given by $\tau:=\sigma+\lambda \sum_{l=1}^{m} \sum_{k=0}^{m_{l}}\left((-1)^{k} u_{l k} / k !\right) \delta^{(k)}\left(x-c_{l}\right)$, where $\lambda$, $\mathfrak{u}_{l k}$, and $c_{l}$ are constants with $c_{i} \neq c_{j}$ for $i \neq j$. That is, $\tau$ is a generalized Uvarov transform of $\sigma$ satisfying $A(x) \tau=A(x) \sigma$, where $A(x)=$ $\prod_{l=1}^{m}\left(x-c_{l}\right)^{m_{l}+1}$. We find necessary and sufficient conditions for $\tau$ to be quasi-definite. We also discuss various properties of monic orthogonal polynomial system $\left\{R_{\mathfrak{n}}(x)\right\}_{\mathfrak{n}=0}^{\infty}$ relative to $\tau$ including two examples.

\section{Introduction}

In the study of Padé approximation (see $[5,10,21])$ of Stieltjes type meromorphic functions

$$
\int_{a}^{b} \frac{d \mu(x)}{z-x}+\sum_{l=1}^{m} \sum_{k=0}^{m_{l}} C_{l k} \frac{k !}{\left(z-c_{l}\right)^{k+1}}
$$

where $-\infty \leq a<b \leq \infty, C_{l k}$ are constants, and $d \mu(x)$ is a positive Stieltjes measure, the denominators $R_{n}(x)$ of the main diagonal sequence of Padé approximants satisfy the orthogonality

$$
\int_{a}^{b} R_{n}(x) \pi(x) d \mu(x)+\sum_{l=1}^{m} \sum_{k=0}^{m_{l}} C_{l k} \partial^{k}\left[\pi R_{n}\right]\left(c_{l}\right)=0, \quad \pi \in \mathbb{P}_{n-1},
$$


where $\mathbb{P}_{n}$ is the space of polynomials of degree $\leq n$ with $\mathbb{P}_{-1}=\{0\}$. That is, $R_{n}(x)(n \geq 0)$ are orthogonal with respect to the measure

$$
d \mu+\sum_{l=1}^{m} \sum_{k=0}^{m_{l}}(-1)^{k} C_{l k} \delta^{(k)}\left(x-c_{l}\right),
$$

which is a point masses perturbation of $d \mu(x)$. Orthogonality to a positive or signed measure perturbed by one or two point masses arises naturally also in orthogonal polynomial eigenfunctions of higher order $(\geq 4)$ ordinary differential equations (see $[14,15,16,19]$ ), which generalize Bochner's classification of classical orthogonal polynomials (see $[6,18])$. On the other hand, many authors have studied various aspects of orthogonal polynomials with respect to various point masses perturbations of positive-definite (see $[1,2,8,14,27,28])$ and quasi-definite (see $[3,4,9,11,12,20,23])$ moment functionals. In this work, we consider the most general such situation. That is, we consider a moment functional $\tau$ given by

$$
\tau:=\sigma+\lambda \sum_{l=1}^{m} \sum_{k=0}^{m_{l}} \frac{(-1)^{k} u_{l k}}{k !} \delta^{(k)}\left(x-c_{l}\right)
$$

where $\sigma$ is a given quasi-definite moment functional, $\lambda, u_{l k}$, and $c_{l}$ are complex numbers with $u_{l, m_{l}} \neq 0$ and $c_{i} \neq c_{j}$ for $i \neq j$, that is, $\tau$ is obtained from $\sigma$ by adding a distribution with finite support. We give necessary and sufficient conditions for $\tau$ to be quasi-definite. When $\tau$ is also quasi-definite, we discuss various properties of orthogonal polynomials $\left\{R_{n}(x)\right\}_{n=0}^{\infty}$ relative to $\tau$ in connection with orthogonal polynomials $\left\{P_{n}(x)\right\}_{n=0}^{\infty}$ relative to $\sigma$. These generalize many previous works in $[4,9,11,12,20,23]$.

\section{Necessary and sufficient conditions}

For any integer $n \geq 0$, let $\mathbb{P}_{n}$ be the space of polynomials of degree $\leq n$ and $\mathbb{P}=\bigcup_{n \geq 0} \mathbb{P}_{n}$. For any $\pi(x)$ in $\mathbb{P}$, let $\operatorname{deg}(\pi)$ be the degree of $\pi(x)$ with the convention that $\operatorname{deg}(0)=-1$. For the moment functionals $\sigma$, $\tau$ (i.e., linear functionals on $\mathbb{P}$ ) (see [7]), $c$ in $\mathbb{C}$, and a polynomial $\phi(x)=\sum_{k=0}^{n} a_{k} x^{k}$, let

$$
\begin{array}{rlrl}
\left\langle\sigma^{\prime}, \pi\right\rangle & :=-\left\langle\sigma, \pi^{\prime}\right\rangle ; & \langle\phi \sigma, \pi\rangle & :=\langle\sigma, \phi \pi\rangle ; \\
\left\langle(x-c)^{-1} \sigma, \pi\right\rangle:=\left\langle\sigma, \theta_{c} \pi\right\rangle ; & \left(\theta_{c} \pi\right)(x):=\frac{\pi(x)-\pi(\mathrm{c})}{x-c} ; \\
(\sigma \phi)(x):=\sum_{k=0}^{n}\left(\sum_{j=k}^{n} a_{j} \sigma_{j k}\right) x^{k} ; & \langle\sigma \tau, \pi\rangle=\langle\sigma, \tau \pi\rangle, \quad \pi \in \mathbb{P} .
\end{array}
$$


We also let

$$
F(\sigma)(z):=\sum_{n=0}^{\infty} \frac{\sigma_{n}}{z^{n+1}}
$$

be the (formal) Stieltjes function of $\sigma$, where $\sigma_{n}:=\left\langle\sigma, x^{n}\right\rangle(n \geq 0)$ are the moments of $\sigma$. Following Zhedanov [29], for any polynomials $A(z), B(z)$, $\mathrm{C}(z), \mathrm{D}(z)$ with no common zero and $|\mathrm{C}|+|\mathrm{D}| \not \equiv 0$, let

$$
S(A, B, C, D) F(\sigma)(z):=\frac{A F(\sigma)+B}{C F(\sigma)+D}
$$

If $S(A, B, C, D) F(\sigma)=F(\tau)$ for some moment functional $\tau$, then we call $\tau$ a rational (resp., linear) spectral transform of $\sigma$ (resp., when $C(z)=0$ ). Then $S(A, B, C, D) F(\sigma)=F(\tau)$ if and only if

$$
\begin{aligned}
x A(x) \sigma & =C(x)(\sigma \tau)+x D(x) \tau \\
\langle\sigma, A\rangle+x\left(\sigma \theta_{0} A\right)(x)+x B(x) & =(\sigma \tau)\left(\theta_{0} C\right)(x)+\langle\tau, D\rangle+x\left(\tau \theta_{0} D\right)(x) .
\end{aligned}
$$

In particular, for any $\mathrm{c}$ and $\beta$ in $\mathbb{C}$, let

$$
\mathrm{U}(\mathrm{c}, \beta) \mathrm{F}(\sigma):=\frac{(z-\mathrm{c}) \mathrm{F}(\sigma)+\beta}{z-\mathrm{c}}
$$

be the Uvarov transform (see $[28,29]$ ) of $F(\sigma)$. Then for any $\left\{c_{i}\right\}_{i=1}^{k}$ and $\left\{\beta_{i}\right\}_{i=1}^{k}$ in $\mathbb{C}$,

$$
F(\tau):=u\left(c_{k}, \beta_{k}\right) \cdots u\left(c_{1}, \beta_{1}\right) F(\sigma)=\frac{A(z) F(\sigma)+B(z)}{A(z)}
$$

where $A(z)=\prod_{i=1}^{k}\left(z-c_{i}\right), B(z)=\sum_{i=1}^{k} \beta_{i} \sum_{\substack{j=1 \\ j \neq i}}^{k}\left(z-c_{j}\right)$, and by (2.4)

$$
A(x) \tau=A(x) \sigma .
$$

In this case, we say that $\tau$ is a generalized Uvarov transform of $\sigma$. Conversely, if (2.7) holds for some polynomial $A(x)(\not \equiv 0)$, then

$$
F(\tau)=\frac{A(z) F(\sigma)+\left(\tau \theta_{0} A\right)(z)-\left(\sigma \theta_{0} A\right)(z)}{A(z)}
$$

and $F(\tau)$ is obtained from $F(\sigma)$ by $\operatorname{deg}(A)$ successive Uvarov transforms (see [29]), that is, $\tau$ is a generalized Uvarov transform of $\sigma$.

In the following, we always assume that $\tau$ is a moment functional given by (1.4), where $\sigma$ is a quasi-definite moment functional. Let $\left\{P_{n}(x)\right\}_{n=0}^{\infty}$ be the monic orthogonal polynomial system (MOPS) relative to $\sigma$ satisfying the 


\section{Generalized Uvarov transforms}

three term recurrence relation

$$
P_{n+1}(x)=\left(x-b_{n}\right) P_{n}(x)-c_{n} P_{n-1}(x), \quad n \geq 0,\left(P_{-1}(x)=0\right) .
$$

Since (1.4) implies (2.7) with $A(x)=\prod_{l=1}^{m}\left(x-c_{l}\right)^{m_{l}+1}$, $\tau$ is a generalized Uvarov transform of $\sigma$. Then our main concern is to find conditions under which a generalized Uvarov transform $\tau$, given by (1.4), of $\sigma$ is also quasidefinite. In other words, we are to solve the division problem (2.7) of the moment functionals.

Let

$$
K_{n}(x, y):=\sum_{j=0}^{n} \frac{P_{j}(x) P_{j}(y)}{\left\langle\sigma, P_{j}^{2}\right\rangle}, \quad n \geq 0
$$

be the $n$th kernel polynomial for $\left\{P_{n}(x)\right\}_{n=0}^{\infty}$ and $K_{n}^{(i, j)}(x, y)=\partial_{x}{ }^{i} \partial_{y}{ }^{j} K_{n}(x, y)$. We need the following lemma which is easy to prove.

Lemma 2.1. Let $\mathrm{V}=\left(\mathrm{x}_{1}, \mathrm{x}_{2}, \ldots, \mathrm{x}_{\mathrm{n}}\right)^{\mathrm{t}}$ and $\mathrm{W}=\left(\mathrm{y}_{1}, \mathrm{y}_{2}, \ldots, \mathrm{y}_{\mathrm{n}}\right)^{\mathrm{t}}$ be two vectors in $\mathbb{C}^{\mathrm{n}}$. Then

$$
\operatorname{det}\left(I_{n}+W^{t}\right)=1+\sum_{j=1}^{n} x_{j} y_{j}, \quad n \geq 1,
$$

where $\mathrm{I}_{\mathrm{n}}$ is the $\mathrm{n} \times \mathrm{n}$ identity matrix.

Theorem 2.2. The moment functional $\tau$ is quasi-definite if and only if $\mathrm{d}_{\mathrm{n}} \neq 0, \mathrm{n} \geq 0$, where $\mathrm{d}_{\mathrm{n}}$ is the determinant of $\left(\sum_{l=1}^{m}\left(m_{l}+1\right)\right) \times$ $\left(\sum_{l=1}^{m}\left(m_{l}+1\right)\right)$ matrix $D_{n}$ :

$$
D_{n}:=\left[A_{t l}(n)\right]_{t, l=1}^{m}, \quad n \geq 0
$$

where

$$
A_{t l}(n)=\left[\delta_{t l} \delta_{k i}+\lambda \sum_{j=0}^{m_{l}-i} \frac{u_{l, i+j}}{i ! j !} K_{n}^{(k, j)}\left(c_{t}, c_{l}\right)\right]_{k=0, i=0}^{m_{t}}
$$

If $\tau$ is quasi-definite, then the MOPS $\left\{\mathrm{R}_{\mathrm{n}}(\mathrm{x})\right\}_{\mathfrak{n}=0}^{\infty}$ relative to $\tau$ is given by

$$
R_{n}(x)=P_{n}(x)-\lambda \sum_{l=1}^{m} \sum_{i=0}^{m_{l}} \sum_{j=0}^{m_{l}-i} \frac{u_{l, i+j}}{i ! j !} K_{n-1}^{(0, j)}\left(x, c_{l}\right) R_{n}^{(i)}\left(c_{l}\right)
$$


where $\left\{R_{n}^{(i)}\left(c_{l}\right)\right\}_{l=1, i=0}^{m} m_{l}$ are given by

$$
D_{n-1}\left[\begin{array}{c}
R_{n}\left(c_{1}\right) \\
R_{n}^{\prime}\left(c_{1}\right) \\
\vdots \\
R_{n}^{\left(m_{1}\right)}\left(c_{1}\right) \\
R_{n}\left(c_{2}\right) \\
\vdots \\
R_{n}^{\left(m_{m}\right)}\left(c_{m}\right)
\end{array}\right]=\left[\begin{array}{c}
P_{n}\left(c_{1}\right) \\
P_{n}^{\prime}\left(c_{1}\right) \\
\vdots \\
P_{n}^{\left(m_{1}\right)}\left(c_{1}\right) \\
P_{n}\left(c_{2}\right) \\
\vdots \\
P_{n}^{\left(m_{m}\right)}\left(c_{m}\right)
\end{array}\right], \quad n \geq 0\left(D_{-1}=I\right) .
$$

Moreover,

$$
\left\langle\tau, R_{n}^{2}\right\rangle=\frac{d_{n}}{d_{n-1}}\left\langle\sigma, P_{n}^{2}\right\rangle, \quad n \geq 0\left(d_{-1}=1\right)
$$

Proof. $(\Rightarrow)$. Assume that $\tau$ is quasi-definite and expand $R_{n}(x)$ as

$$
R_{n}(x)=P_{n}(x)+\sum_{j=0}^{n-1} C_{n j} P_{j}(x), \quad n \geq 1,
$$

where $C_{n j}=\left\langle\sigma, R_{n} P_{j}\right\rangle /\left\langle\sigma, P_{j}^{2}\right\rangle$, with $0 \leq j \leq n-1$. Here,

$$
\begin{aligned}
\left\langle\sigma, R_{n} P_{j}\right\rangle & =\left\langle\tau-\lambda \sum_{l=1}^{m} \sum_{k=0}^{m_{l}} \frac{(-1)^{k} u_{l k}}{k !} \delta^{(k)}\left(x-c_{l}\right), R_{n} P_{j}\right\rangle \\
& =-\lambda \sum_{l=1}^{m} \sum_{k=0}^{m_{l}} \frac{u_{l k}}{k !} \sum_{i=0}^{k}\left(\begin{array}{l}
k \\
i
\end{array}\right) R_{n}^{(i)}\left(c_{l}\right) P_{j}^{(k-i)}\left(c_{l}\right)
\end{aligned}
$$

so that

$$
\begin{aligned}
R_{n}(x) & =P_{n}(x)-\lambda \sum_{j=0}^{n-1} \frac{P_{j}(x)}{\left\langle\sigma, P_{j}^{2}\right\rangle} \sum_{l=1}^{m} \sum_{k=0}^{m_{l}} \frac{u_{l k}}{k !} \sum_{i=0}^{k}\left(\begin{array}{l}
k \\
i
\end{array}\right) R_{n}^{(i)}\left(c_{l}\right) P_{j}^{(k-i)}\left(c_{l}\right) \\
& =P_{n}(x)-\lambda \sum_{l=1}^{m} \sum_{k=0}^{m_{l}} \frac{u_{l k}}{k !} \sum_{i=0}^{k}\left(\begin{array}{l}
k \\
i
\end{array}\right) R_{n}^{(i)}\left(c_{l}\right) K_{n-1}^{(0, k-i)}\left(x, c_{l}\right) \\
& =P_{n}(x)-\lambda \sum_{l=1}^{m} \sum_{i=0}^{m_{l}} \sum_{j=0}^{m_{l}-i} \frac{u_{l, i+j}}{i ! j !} K_{n-1}^{(0, j)}\left(x, c_{l}\right) R_{n}^{(i)}\left(c_{l}\right) .
\end{aligned}
$$

Hence, we have (2.14). Set the matrices $B_{l}$ and $E_{l}$ to be

$$
B_{l}=\left[\begin{array}{c}
R_{n}\left(c_{l}\right) \\
R_{n}^{\prime}\left(c_{l}\right) \\
\vdots \\
R_{n}^{\left(m_{l}\right)}\left(c_{l}\right)
\end{array}\right], \quad E_{l}=\left[\begin{array}{c}
P_{n}\left(c_{l}\right) \\
P_{n}^{\prime}\left(c_{l}\right) \\
\vdots \\
P_{n}^{\left(m_{l}\right)}\left(c_{l}\right)
\end{array}\right], \quad 1 \leq l \leq m .
$$




\section{Generalized Uvarov transforms}

Then,

$$
\left[\begin{array}{c}
E_{1} \\
E_{2} \\
\vdots \\
E_{m}
\end{array}\right]=\left[A_{t l}(n-1)\right]_{t, l=1}^{m}\left[\begin{array}{c}
B_{1} \\
B_{2} \\
\vdots \\
B_{m}
\end{array}\right]
$$

which gives (2.15). Now,

$$
\begin{aligned}
& D_{n}=\left[A_{t l}(n)\right]_{t, l=1}^{m} \\
& =D_{n-1}+\frac{\lambda}{\left\langle\sigma, P_{n}^{2}\right\rangle}\left[\left[\sum_{j=0}^{m_{l}-i} \frac{u_{l, i+j}}{i ! j !} P_{n}^{(j)}\left(c_{l}\right) P_{n}^{(k)}\left(c_{t}\right)\right]_{k=0, i=0}^{m_{t}}\right]_{t, l=1}^{m_{l}} \\
& =D_{n-1}+\frac{\lambda}{\left\langle\sigma, P_{n}^{2}\right\rangle}\left[\begin{array}{c}
E_{1} \\
E_{2} \\
\vdots \\
E_{m}
\end{array}\right]\left[\sum_{j=0}^{m_{1}} \frac{u_{1 j}}{j !} P_{n}^{(j)}\left(c_{1}\right), \sum_{j=0}^{m_{1}-1} \frac{u_{1, j+1}}{j !} P_{n}^{(j)}\left(c_{1}\right), \ldots,\right. \\
& \frac{u_{1, m_{1}}}{m_{1} !} P_{n}\left(c_{1}\right), \sum_{j=0}^{m_{2}} \frac{u_{2 j}}{j !} P_{n}^{(j)}\left(c_{2}\right), \ldots \\
& \left.\frac{u_{m, m_{m}}}{m_{m} !} P_{n}\left(c_{m}\right)\right] \\
& =D_{n-1}\left[I+\frac{\lambda}{\left\langle\sigma, P_{n}^{2}\right\rangle}\left[\begin{array}{c}
B_{1} \\
B_{2} \\
\vdots \\
B_{m}
\end{array}\right]\left[\sum_{j=0}^{m_{1}} \frac{u_{1 j}}{j !} P_{n}^{(j)}\left(c_{1}\right), \sum_{j=0}^{m_{1}-1} \frac{u_{1, j+1}}{j !} P_{n}^{(j)}\left(c_{1}\right), \ldots,\right.\right. \\
& \frac{u_{1, m_{1}}}{m_{1} !} P_{n}\left(c_{1}\right), \sum_{j=0}^{m_{2}} \frac{u_{2 j}}{j !} P_{n}^{(j)}\left(c_{2}\right), \ldots \\
& \left.\frac{u_{m, m_{m}}}{m_{m} !} P_{n}\left(c_{m}\right)\right]
\end{aligned}
$$


so that

$$
d_{n}=d_{n-1}\left(1+\frac{\lambda}{\left\langle\sigma, P_{n}^{2}\right\rangle} \sum_{l=1}^{m} \sum_{i=0}^{m_{l}} \sum_{j=0}^{m_{l}-i} \frac{u_{l, i+j}}{i ! j !} P_{n}^{(j)}\left(c_{l}\right) R_{n}^{(i)}\left(c_{l}\right)\right)
$$

by Lemma 2.1. On the other hand,

$$
\begin{aligned}
\left\langle\tau, R_{n}^{2}\right\rangle & =\left\langle\tau, R_{n} P_{n}\right\rangle \\
& =\left\langle\sigma, R_{n} P_{n}\right\rangle+\lambda\left\langle\sum_{l=1}^{m} \sum_{k=0}^{m_{l}} \frac{(-1)^{k} u_{l k}}{k !} \delta^{(k)}\left(x-c_{l}\right), R_{n} P_{n}\right\rangle \\
& =\left\langle\sigma, P_{n}^{2}\right\rangle+\lambda \sum_{l=1}^{m} \sum_{k=0}^{m_{l}} \frac{u_{l k}}{k !} \sum_{j=0}^{k}\left(\begin{array}{l}
k \\
j
\end{array}\right) R_{n}^{(j)}\left(c_{l}\right) P_{n}^{(k-j)}\left(c_{l}\right) \\
& =\left\langle\sigma, P_{n}^{2}\right\rangle+\lambda \sum_{l=1}^{m} \sum_{j=0}^{m_{l}} \sum_{k=j}^{m_{l}} \frac{u_{l k}}{k !}\left(\begin{array}{l}
k \\
j
\end{array}\right) R_{n}^{(j)}\left(c_{l}\right) P_{n}^{(k-j)}\left(c_{l}\right)
\end{aligned}
$$

so that

$$
\left\langle\tau, R_{n}^{2}\right\rangle=\left\langle\sigma, P_{n}^{2}\right\rangle+\lambda \sum_{l=1}^{m} \sum_{j=0}^{m_{l}} \sum_{k=0}^{m_{l}-j} \frac{u_{l, j+k}}{j ! k !} R_{n}^{(j)}\left(c_{l}\right) P_{n}^{(k)}\left(c_{l}\right) .
$$

Hence, from (2.23) and (2.25), we have

$$
\left\langle\sigma, P_{n}^{2}\right\rangle d_{n}=d_{n-1}\left\langle\tau, R_{n}^{2}\right\rangle, \quad n \geq 0
$$

Note that (2.26) also holds for $n=0$ if we take $d_{-1}=1$. Hence, $d_{n} \neq 0$, $n \geq 0$ inductively and we have (2.16).

$(\Leftarrow)$. Assume that $d_{n} \neq 0$, with $n \geq 0$ and define $\left\{R_{n}(x)\right\}_{n=0}^{\infty}$ by (2.14). Then we have, by (2.14) and (2.23), 
76 Generalized Uvarov transforms

$$
\begin{aligned}
& \left\langle\tau, R_{n} P_{r}\right\rangle=\left\langle\sigma, R_{n} P_{r}\right\rangle+\lambda\left\langle\sum_{l=1}^{m} \sum_{k=0}^{m_{l}} \frac{(-1)^{k} u_{l k}}{k !} \delta^{(k)}\left(x-c_{l}\right), R_{n} P_{r}\right\rangle \\
& =\left\langle\sigma, R_{n} P_{r}\right\rangle+\lambda \sum_{l=1}^{m} \sum_{k=0}^{m_{l}} \frac{u_{l k}}{k !} \sum_{j=0}^{k}\left(\begin{array}{l}
k \\
j
\end{array}\right) R_{n}^{(j)}\left(c_{l}\right) P_{r}^{(k-j)}\left(c_{l}\right) \\
& =\left\langle\sigma, P_{n} P_{r}\right\rangle-\lambda \sum_{l=1}^{m} \sum_{j=0}^{m_{l}} \sum_{k=0}^{m_{l}-j} \frac{u_{l, j+k}}{j ! k !} R_{n}^{(j)}\left(c_{l}\right)\left\langle\sigma, K_{n-1}^{(0, k)}\left(x, c_{l}\right) P_{r}(x)\right\rangle \\
& +\lambda \sum_{l=1}^{m} \sum_{j=0}^{m_{l}} \sum_{k=0}^{m_{l}-j} \frac{u_{l, j+k}}{j ! k !} R_{n}^{(j)}\left(c_{l}\right) P_{r}^{(k)}\left(c_{l}\right) \\
& =\left\langle\sigma, P_{n} P_{r}\right\rangle-\lambda \sum_{l=1}^{m} \sum_{j=0}^{m_{l}} \sum_{k=0}^{m_{l}-j} \frac{u_{l, j+k}}{j ! k !} R_{n}^{(j)}\left(c_{l}\right) P_{r}^{(k)}\left(c_{l}\right)\left(1-\delta_{n r}\right) \\
& +\lambda \sum_{l=1}^{m} \sum_{j=0}^{m_{l}} \sum_{k=0}^{m_{l}-j} \frac{u_{l, j+k}}{j ! k !} R_{n}^{(j)}\left(c_{l}\right) P_{r}^{(k)}\left(c_{l}\right) \\
& =\left\{\begin{array}{l}
0, \quad 0 \leq r \leq n-1, \\
\left\langle\sigma, P_{n}^{2}\right\rangle+\lambda \sum_{l=1}^{m} \sum_{j=0}^{m_{l}} \sum_{k=0}^{m_{l}-j} \frac{u_{l, j+k}}{j ! k !} P_{n}^{(k)}\left(c_{l}\right) R_{n}^{(j)}\left(c_{l}\right), \quad r=n,
\end{array}\right. \\
& =\left\{\begin{array}{l}
0, \quad 0 \leq r \leq n-1, \\
\frac{d_{n}}{d_{n-1}}\left\langle\sigma, P_{n}^{2}\right\rangle \neq 0, \quad r=n,
\end{array}\right.
\end{aligned}
$$

since $\left\langle\sigma, K_{n-1}^{(0, k)}\left(x, c_{l}\right) P_{r}(x)\right\rangle=P_{r}^{(k)}\left(c_{l}\right)\left(1-\delta_{n r}\right)$. Hence,

$$
\left\langle\tau, R_{n} R_{m}\right\rangle=\left\{\begin{array}{l}
0, \quad \text { if } 0 \leq m \leq n-1 \\
\left\langle\tau, R_{n} P_{n}\right\rangle \neq 0, \quad \text { if } m=n
\end{array}\right.
$$

so that $\left\{R_{n}(x)\right\}_{n=0}^{\infty}$ is the MOPS relative to $\tau$ and so $\tau$ is also quasi-definite.

General division problems of moment functionals

$$
\mathrm{D}(x) \tau=A(x) \sigma
$$

is handled in [17], when $\mathrm{D}(\mathrm{x})$ and $\mathrm{A}(\mathrm{x})$ have no common zero. Theorem 2.2 includes the following as special cases.

- $\mathrm{m}=1, \mathrm{~m}_{1}=0$ : Marcellán and Maroni [23],

- $\mathrm{m}=2, \mathrm{~m}_{1}=\mathrm{m}_{2}=0$ : Draïdi and Maroni [9], Kwon and Park [20], 
- $m=1, m_{1}=1$ : Belmehdi and Marcellán [4],

- $\mathrm{m}=1$ : Kim, Kwon, and Park [12].

Some other special cases where $\sigma$ is a classical moment functional were handled in $[2,1,3,8,11,14]$.

From now on, we always assume that $d_{n} \neq 0$, with $n \geq 0$, so that $\tau$ is also quasi-definite.

Theorem 2.3. For the MOPS $\left\{\mathrm{R}_{\mathrm{n}}(\mathrm{x})\right\}_{\mathfrak{n}=0}^{\infty}$ relative to $\tau$, we have

(i) (the three-term recurrence relation)

$$
R_{n+1}(x)=\left(x-\beta_{n}\right) R_{n}(x)-\gamma_{n} R_{n-1}(x), \quad n \geq 0,
$$

where

$$
\begin{aligned}
\beta_{n}= & b_{n}+\frac{\lambda}{\left\langle\sigma, P_{n}^{2}\right\rangle} \sum_{l=1}^{m} \sum_{i=0}^{m_{l}} \sum_{j=0}^{m_{l}-i} \frac{u_{l, i+j}}{i ! j !} \\
& \times\left\{P_{n}^{(j)}\left(c_{l}\right) R_{n+1}^{(i)}\left(c_{l}\right)-P_{n-1}^{(j)}\left(c_{l}\right) R_{n}^{(i)}\left(c_{l}\right)\right\} \quad(n \geq 0), \\
& \gamma_{n}=\frac{d_{n} d_{n-2}}{d_{n-1}^{2}} c_{n} \quad(n \geq 1) .
\end{aligned}
$$

(ii) (the quasi-orthogonality)

$$
\prod_{l=1}^{m}\left(x-c_{l}\right)^{m_{l}+1} R_{n}(x)=\sum_{j=n-r}^{n+r} C_{n j} P_{j}(x), \quad n \geq r,
$$

where $r=\sum_{l=1}^{m}\left(m_{l}+1\right), C_{n, n-r} \neq 0$, and

$$
\begin{aligned}
C_{n j} & =\frac{\left\langle\sigma, \prod_{l=1}^{m}\left(x-c_{l}\right)^{m_{l}+1} R_{n} P_{j}\right\rangle}{\left\langle\sigma, P_{j}^{2}\right\rangle} \\
& =\frac{\left\langle\tau, \prod_{l=1}^{m}\left(x-c_{l}\right)^{m_{l}+1} R_{n} P_{j}\right\rangle}{\left\langle\sigma, P_{j}^{2}\right\rangle}, \text { where } n-r \leq j \leq n+r .
\end{aligned}
$$

Proof. For (i), by (2.14), we can rewrite (2.30) as

$$
\begin{aligned}
P_{n+1}(x) & -\lambda \sum_{l=1}^{m} \sum_{i=0}^{m_{l}} \sum_{j=0}^{m_{l}-i} \frac{u_{l, i+j}}{i ! j !} K_{n}^{(0, j)}\left(x, c_{l}\right) R_{n+1}^{(i)}\left(c_{l}\right) \\
= & \left(x-\beta_{n}\right)\left\{P_{n}(x)-\lambda \sum_{l=1}^{m} \sum_{i=0}^{m_{l}} \sum_{j=0}^{m_{l}-i} \frac{u_{l, i+j}}{i ! j !} K_{n-1}^{(0, j)}\left(x, c_{l}\right) R_{n}^{(i)}\left(c_{l}\right)\right\} \\
& -\gamma_{n}\left\{P_{n-1}(x)-\lambda \sum_{l=1}^{m} \sum_{i=0}^{m_{l}} \sum_{j=0}^{m_{l}-i} \frac{u_{l, i+j}}{i ! j !} K_{n-2}^{(0, j)}\left(x, c_{l}\right) R_{n-1}^{(i)}\left(c_{l}\right)\right\} .
\end{aligned}
$$


After multiplying (2.35) by $P_{n}(x)$ and applying $\sigma$, we have

$$
\begin{aligned}
& -\lambda \sum_{l=1}^{m} \sum_{i=0}^{m_{l}} \sum_{j=0}^{m_{l}-i} \frac{u_{l, i+j}}{i ! j !} P_{n}^{(j)}\left(c_{l}\right) R_{n+1}^{(i)}\left(c_{l}\right) \\
& \quad=\left(b_{n}-\beta_{n}\right)\left\langle\sigma, P_{n}^{2}\right\rangle-\lambda \sum_{l=1}^{m} \sum_{i=0}^{m_{l}} \sum_{j=0}^{m_{l}-i} \frac{u_{l, i+j}}{i ! j !} P_{n-1}^{(j)}\left(c_{l}\right) R_{n}^{(i)}\left(c_{l}\right) .
\end{aligned}
$$

Hence, we have (2.31) and by (2.16)

$$
\gamma_{n}=\frac{\left\langle\tau, R_{n}^{2}\right\rangle}{\left\langle\tau, R_{n-1}^{2}\right\rangle}=\frac{d_{n} d_{n-2}}{d_{n-1}^{2}} c_{n} \quad(n \geq 1) .
$$

For (ii), $\prod_{l=1}^{m}\left(x-c_{l}\right)^{m_{l}+1} R_{n}(x)=\sum_{j=0}^{n+r} C_{n j} P_{j}(x)$, where $r=\sum_{l=1}^{m}\left(m_{l}+1\right)$ and

$$
\begin{aligned}
C_{n k}\left\langle\sigma, P_{k}^{2}\right\rangle & =\left\langle\sigma, \prod_{l=1}^{m}\left(x-c_{l}\right)^{m_{l}+1} R_{n}(x) P_{k}(x)\right\rangle \\
& =\left\langle\prod_{l=1}^{m}\left(x-c_{l}\right)^{m_{l}+1} \tau, R_{n}(x) P_{k}(x)\right\rangle \\
& =\left\langle\tau, \prod_{l=1}^{m}\left(x-c_{l}\right)^{m_{l}+1} R_{n}(x) P_{k}(x)\right\rangle=0, \quad \text { if } r+k<n .
\end{aligned}
$$

Hence, $C_{n k}=0,0 \leq k \leq n-r-1$ and $C_{n, n-r} \neq 0$ so that we have (2.33) and (2.34).

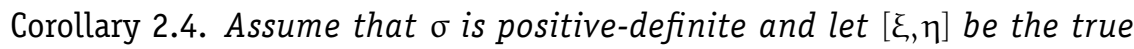
interval of the orthogonality of $\sigma$. Then

(i) $\prod_{l=1}^{m}\left(x-c_{l}\right)^{m_{l}+1} R_{n}(x)$ has at least $n-r$ distinct nodal zeros (i.e., zeros of odd multiplicities) in ( $\xi, \eta)$.

(ii) $R_{n}(x)$ has at least $n-r-m$ distinct nodal zeros in $(\xi, \eta)$.

If furthermore $m_{l}(1 \leq l \leq m)$ are odd or $\xi \geq c_{l}(1 \leq l \leq m)$, then

(iii) $R_{n}(x)$ has at least $n-r$ distinct nodal zeros in $(\xi, \eta)$.

Proof. (i) and (ii) are trivial by (2.33).

For (iii), assume that $m_{l}(1 \leq l \leq m)$ are odd. Then $\tilde{\sigma}:=\prod_{l=1}^{m}(x-$ $\left.c_{l}\right)^{m_{l}+1} \sigma$ is also positive-definite on $[\xi, \eta]$. Let $\left\{\tilde{P}_{n}(x)\right\}_{n=0}^{\infty}$ be the MOPS relative to $\tilde{\sigma}$. Then we may write $R_{n}(x)=\sum_{j=0}^{n} \tilde{C}_{n j} \tilde{P}_{j}(x)$, where

$$
\begin{aligned}
\tilde{C}_{n k}\left\langle\tilde{\sigma}, \tilde{P}_{k}^{2}\right\rangle=\left\langle\tilde{\sigma}, R_{n} \tilde{P}_{k}\right\rangle & =\left\langle\prod_{l=1}^{m}\left(x-c_{l}\right)^{m_{l}+1} \tau, R_{n} \tilde{P}_{k}\right\rangle \\
& =\left\langle\tau, \prod_{l=1}^{m}\left(x-c_{l}\right)^{m_{l}+1} R_{n} \tilde{P}_{k}\right\rangle .
\end{aligned}
$$


Hence, $\tilde{C}_{n k}=0,0 \leq k \leq n-r-1$ so that $R_{n}(x)=\sum_{j=n-r}^{n} \tilde{C}_{n j} \tilde{P}_{j}(x)$. Hence, $R_{n}(x)$ has at least $n-r$ distinct nodal zeros in $(\xi, \eta)$. In case $\xi \geq c_{l}(1 \leq$

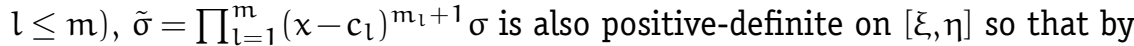
the same reasoning as above $R_{n}(x)$ has at least $n-r$ distinct nodal zeros in $(\xi, \eta)$.

Theorem 2.5. For any polynomial $\mathrm{p}(\mathrm{x})$ of degree at most $\mathrm{n}$, we have

$$
\left\langle\tau, \mathrm{L}_{\mathrm{n}}^{(0, k)}(x, y) p(x)\right\rangle=\mathrm{p}^{(\mathrm{k})}(\mathrm{y}),
$$

where $L_{n}(x, y)=\sum_{i=0}^{n} R_{i}(x) R_{i}(y) /\left\langle\tau, R_{i}^{2}\right\rangle, n \geq 0$, is the $n$th kernel polynomial for $\left\{R_{n}(x)\right\}_{n=0}^{\infty}$ and

$$
L_{n}(x, y)=K_{n}(x, y)-\frac{\lambda}{d_{n}} \sum_{l=1}^{m} \sum_{i=0}^{m_{l}}\left|D_{n}^{u}\right| \sum_{j=0}^{m_{l}-i} \frac{u_{l, i+j}}{i ! j !} K_{n}^{(0, j)}\left(x, c_{l}\right),
$$

where $u=\sum_{k=1}^{l-1}\left(m_{k}+1\right)+(i+1)$ and $D_{n}^{i}$ is the matrix obtained from $\mathrm{D}_{\mathrm{n}}$ by replacing the ith column of $\mathrm{D}_{\mathrm{n}}$ by

$$
\begin{aligned}
& {\left[K_{n}\left(c_{1}, y\right), K_{n}^{(1,0)}\left(c_{1}, y\right), \ldots, K_{n}^{\left(m_{1}, 0\right)}\left(c_{1}, y\right),\right.} \\
& \left.K_{n}\left(c_{2}, y\right), K_{n}^{(1,0)}\left(c_{2}, y\right), \ldots, K_{n}^{\left(m_{m}, 0\right)}\left(c_{m}, y\right)\right]^{\top} .
\end{aligned}
$$

Proof. If $\operatorname{deg}(p) \leq n$, then $p(x)=\sum_{i=0}^{n}\left(\left\langle\tau, p R_{i}\right\rangle /\left\langle\tau, R_{i}^{2}\right\rangle\right) R_{i}(x)$ so that

$$
\begin{aligned}
\left\langle\tau, \mathrm{L}_{n}^{(0, k)}(x, y) p(x)\right\rangle & =\sum_{i=0}^{n} \frac{\left\langle\tau, p R_{i}\right\rangle}{\left\langle\tau, R_{i}^{2}\right\rangle}\left\langle\tau, L_{n}^{(0, k)}(x, y) R_{i}(x)\right\rangle \\
& =\sum_{i=0}^{n} \frac{\left\langle\tau, p R_{i}\right\rangle}{\left\langle\tau, R_{i}^{2}\right\rangle} \sum_{j=0}^{n} \frac{R_{j}^{(k)}(y)}{\left\langle\tau, R_{j}^{2}\right\rangle}\left\langle\tau, R_{j}(x) R_{i}(x)\right\rangle \\
& =\sum_{i=0}^{n} \frac{\left\langle\tau, p R_{i}\right\rangle}{\left\langle\tau, R_{i}^{2}\right\rangle} R_{i}^{(k)}(y)=p^{(k)}(y) .
\end{aligned}
$$

Expand $L_{n}(x, y)$ as $L_{n}(x, y)=\sum_{j=0}^{n} a_{n j}(y) P_{j}(x)$, where

$$
\begin{aligned}
a_{n j}(y)= & \frac{\left\langle\sigma, L_{n}(x, y) P_{j}(x)\right\rangle}{\left\langle\sigma, P_{j}^{2}\right\rangle} \\
= & \frac{\left\langle\tau, L_{n}(x, y) P_{j}(x)\right\rangle}{\left\langle\sigma, P_{j}^{2}\right\rangle}-\frac{\lambda}{\left\langle\sigma, P_{j}^{2}\right\rangle} \\
& \times \sum_{l=1}^{m} \sum_{k=0}^{m_{l}} \frac{(-1)^{k} u_{l k}}{k !}\left\langle\delta^{(k)}\left(x-c_{l}\right), L_{n}(x, y) P_{j}(x)\right\rangle \\
= & \frac{P_{j}(y)}{\left\langle\sigma, P_{j}^{2}\right\rangle}-\frac{\lambda}{\left\langle\sigma, P_{j}^{2}\right\rangle} \sum_{l=1}^{m} \sum_{k=0}^{m_{l}} \frac{u_{l k}}{k !} \sum_{i=0}^{k}\left(\begin{array}{l}
k \\
i
\end{array}\right) L_{n}^{(i, 0)}\left(c_{l}, y\right) P_{j}^{(k-i)}\left(c_{l}\right)
\end{aligned}
$$


by (2.40). Hence

$$
\begin{aligned}
\mathrm{L}_{n}(x, y) & =\mathrm{K}_{n}(x, y)-\lambda \sum_{l=1}^{m} \sum_{k=0}^{m_{l}} \frac{u_{l k}}{k !} \sum_{i=0}^{k}\left(\begin{array}{l}
k \\
i
\end{array}\right) L_{n}^{(i, 0)}\left(c_{l}, y\right) K_{n}^{(0, k-i)}\left(x, c_{l}\right) \\
& =K_{n}(x, y)-\lambda \sum_{l=1}^{m} \sum_{i=0}^{m_{l}} \sum_{j=0}^{m_{l}-i} \frac{u_{l, i+j}}{i ! j !} K_{n}^{(0, j)}\left(x, c_{l}\right) L_{n}^{(i, 0)}\left(c_{l}, y\right)
\end{aligned}
$$

and so

$$
\begin{aligned}
D_{n}\left[L_{n}\left(c_{1}, y\right), L_{n}^{(1,0)}\left(c_{1}, y\right), \ldots, L_{n}^{\left(m_{1}, 0\right)}\left(c_{1}, y\right),\right. \\
\left.L_{n}\left(c_{2}, y\right), \ldots, L_{n}^{\left(m_{m}, 0\right)}\left(c_{m}, y\right)\right]^{\top} \\
=\left[K_{n}\left(c_{1}, y\right), K_{n}^{(1,0)}\left(c_{1}, y\right), \ldots, K_{n}^{\left(m_{1}, 0\right)}\left(c_{1}, y\right),\right. \\
\left.\quad K_{n}\left(c_{2}, y\right), \ldots, K_{n}^{\left(m_{m}, 0\right)}\left(c_{m}, y\right)\right]^{\top} .
\end{aligned}
$$

Hence, we have (2.41) from (2.45) and (2.46).

\section{Semi-classical case}

Since $\tau$ is a linear spectral transform (see [29]) of $\sigma$, if $\sigma$ is a LaguerreHahn form (see [22]) or a semi-classical form (see [24]) or a second degree form (see [26]), then so is $\tau$. Here, we consider the semi-classical case more closely.

Definition 3.1 (see Maroni [24]). A moment functional $\sigma$ is said to be semiclassical if $\sigma$ is quasi-definite and satisfies a Pearson type functional equation

$$
(\phi(x) \sigma)^{\prime}-\psi(x) \sigma=0
$$

for some polynomials $\phi(x)$ and $\psi(x)$ with $\operatorname{deg}(\phi) \geq 0$ and $\operatorname{deg}(\psi) \geq 1$.

For a semi-classical moment functional $\sigma$, we call

$$
s:=\min \max (\operatorname{deg}(\phi)-2, \operatorname{deg}(\psi)-1)
$$

the class number of $\sigma$, where the minimum is taken over all pairs $(\phi, \psi)$ of polynomials satisfying (3.1). We then call the MOPS $\left\{\mathrm{P}_{\mathfrak{n}}(x)\right\}_{\mathfrak{n}=0}^{\infty}$ relative to $\sigma$ a semi-classical OPS (SCOPS) of class s.

From now on, we assume that $\sigma$ is a semi-classical moment functional satisfying (3.1) and set $s:=\max (\operatorname{deg}(\phi)-2, \operatorname{deg}(\psi)-1)$. Then $\tau$ satisfies the functional equation

$$
(\mathrm{T}(\mathrm{x}) \phi(\mathrm{x}) \tau)^{\prime}=\left(\mathrm{T}^{\prime}(\mathrm{x}) \phi(\mathrm{x})+\mathrm{T}(\mathrm{x}) \psi(\mathrm{x})\right) \tau
$$


where

$$
\mathrm{T}(\mathrm{x})=\prod_{\mathrm{l}=1}^{\mathrm{m}}\left(\mathrm{x}-\mathrm{c}_{\mathrm{l}}\right)^{\mathrm{m}_{\mathrm{l}}+2} .
$$

We now determine the class number of $\tau$. By (3.3), if $\sigma$ is of class $s$, then $\tau$ is of class $\leq s+\sum_{l=1}^{m}\left(m_{l}+2\right)$.

Lemma 3.2 (see [25]). The semi-classical moment functional $\sigma$ satisfying (3.1) is of class s if and only if for any zero $c$ of $\phi(x)$,

$$
\mathbb{N}(\sigma ; c):=\left|r_{c}\right|+\left|\left\langle\sigma, q_{c}(x)\right\rangle\right| \neq 0,
$$

where $\phi(x)=(x-c) \phi_{c}(x)$ and $\phi_{c}(x)-\psi(x)=(x-c) q_{c}(x)+r_{c}$.

Theorem 3.3. Assume that $\sigma$ is of class $s=\max (\operatorname{deg}(\phi)-2, \operatorname{deg}(\psi)-1)$. Then $\tau$ is of class $\mathrm{s}+\sum_{\mathrm{l}=1}^{\mathrm{m}}\left(\mathrm{m}_{\mathrm{l}}+2\right)$ if $\phi\left(\mathrm{c}_{\mathrm{l}}\right) \neq 0,1 \leq \mathrm{l} \leq \mathrm{m}$.

Proof. Assume $\phi\left(c_{l}\right) \neq 0,1 \leq l \leq m$. Let $\tilde{\phi}(x)=T(x) \phi(x)$ and $\tilde{\psi}(x)=$ $\mathrm{T}^{\prime}(x) \phi(x)+\mathrm{T}(x) \psi(x)$. For any zero $c$ of $\tilde{\phi}(x)$, let $\tilde{\phi}(x)=(x-c) \tilde{\phi}_{c}(x)$ and $\tilde{\phi}_{c}(x)-\tilde{\psi}(x)=(x-c) \tilde{q}_{c}(x)+\tilde{r}_{c}$. Then either $c=c_{t}(1 \leq t \leq m)$ or $\phi(c)=0$.

If $c=c_{t}(1 \leq t \leq m)$, then

$$
\tilde{\phi}_{c}(x)-\tilde{\psi}(x)=\frac{T(x) \phi(x)}{x-c_{t}}-T^{\prime}(x) \phi(x)-T(x) \psi(x)=\left(x-c_{t}\right) \tilde{q}_{c}(x) .
$$

Hence, $\tilde{\mathrm{r}}_{\mathrm{c}}=0$ but

$$
\begin{aligned}
& \left\langle\tau, \tilde{\mathrm{q}}_{\mathrm{c}}(x)\right\rangle=\left\langle\sigma+\lambda \sum_{\mathrm{l}=1}^{\mathrm{m}} \sum_{k=0}^{\mathrm{m}_{\mathrm{l}}} \frac{(-1)^{\mathrm{k}} \mathrm{u}_{\mathrm{lk}}}{\mathrm{k} !} \delta^{(k)}\left(x-\mathrm{c}_{l}\right), \tilde{\mathrm{q}}_{\mathrm{c}}(x)\right\rangle \\
& =\left\langle\sigma+\lambda \sum_{l=1}^{m} \sum_{k=0}^{m_{l}} \frac{(-1)^{k} u_{l k}}{k !} \delta^{(k)}\left(x-c_{l}\right)\right. \text {, } \\
& \left.\frac{\mathrm{T}(x) \phi(x)}{\left(x-c_{t}\right)^{2}}-\frac{\mathrm{T}^{\prime}(x) \phi(x)+\mathrm{T}(x) \psi(x)}{x-c_{t}}\right\rangle \\
& =\left\langle(\phi \sigma)^{\prime}-\psi \sigma, \frac{T(x)}{x-c_{t}}\right\rangle \\
& +\left\langle\lambda \sum_{l=1}^{m} \sum_{k=0}^{m_{l}} \frac{(-1)^{k} u_{l k}}{k !} \delta^{(k)}\left(x-c_{l}\right),\right. \\
& \left.\frac{\mathrm{T}(\mathrm{x}) \phi(\mathrm{x})}{\left(\mathrm{x}-\mathrm{c}_{\mathrm{t}}\right)^{2}}-\frac{\mathrm{T}^{\prime}(\mathrm{x}) \phi(\mathrm{x})+\mathrm{T}(\mathrm{x}) \psi(\mathrm{x})}{\mathrm{x}-\mathrm{c}_{\mathrm{t}}}\right\rangle \\
& =-\lambda u_{t, m_{t}}\left(m_{t}+1\right) \prod_{\substack{l=1 \\
l \neq t}}^{m}\left(c_{t}-c_{l}\right) \phi\left(c_{t}\right) \neq 0,
\end{aligned}
$$

so that $\mathbb{N}(\tau, c) \neq 0$. 


\section{Generalized Uvarov transforms}

If $c \neq c_{t}(1 \leq t \leq m)$, then $\phi(c)=0, \tilde{\phi}_{c}(x)=T(x) \phi_{c}(x)$, and

$$
\tilde{\phi}_{c}(x)-\tilde{\psi}(x)=\mathrm{T}(x) \phi_{c}(x)-T^{\prime}(x) \phi(x)-T(x) \psi(x) .
$$

Hence, $\tilde{r}_{c}=\mathrm{T}(\mathrm{c}) \phi_{\mathrm{c}}(\mathrm{c})-\mathrm{T}(\mathrm{c}) \psi(\mathrm{c})=\mathrm{T}(\mathrm{c}) \mathrm{r}_{\mathrm{c}}$. If $\mathrm{r}_{\mathrm{c}} \neq 0$, then $\tilde{r}_{\mathrm{c}} \neq 0$ so that $\mathbb{N}(\tau ; c) \neq 0$.

If $r_{c}=0$, then $\left\langle\sigma, q_{c}(x)\right\rangle \neq 0$ and $\tilde{r}_{c}=0$ so that

$$
\tilde{\mathrm{q}}_{\mathrm{c}}(x)=\mathrm{T}(x) \mathrm{q}_{\mathrm{c}}(x)-\mathrm{T}^{\prime}(x) \phi_{\mathrm{c}}(x) .
$$

Then

$$
\left\langle\tau, \tilde{\mathrm{q}}_{\mathrm{c}}(\mathrm{x})\right\rangle=\left\langle\sigma, \tilde{\mathrm{q}}_{\mathrm{c}}(\mathrm{x})\right\rangle=\left\langle\sigma, \mathrm{T}(\mathrm{x}) \mathrm{q}_{\mathrm{c}}(\mathrm{x})-\mathrm{T}^{\prime}(\mathrm{x}) \phi_{\mathrm{c}}(\mathrm{x})\right\rangle .
$$

Set $Q_{1}(x), Q_{2}(x), Q_{3}(x)$, and $r_{1}, r_{2}, r_{3}$ to be

$$
\begin{aligned}
T(x) & =(x-c) Q_{1}(x)+r_{1} ; \\
T^{\prime}(x) & =(x-c) Q_{2}(x)+r_{2} ; \\
Q_{1}(x) & =(x-c) Q_{3}(x)+r_{3} .
\end{aligned}
$$

Then $\mathrm{Q}_{2}(\mathrm{x})=\mathrm{Q}_{1}^{\prime}(\mathrm{x})+\mathrm{Q}_{3}(\mathrm{x})$ and $\mathrm{r}_{2}=\mathrm{r}_{3}=\mathrm{Q}_{1}(\mathrm{c})$.

Hence,

$$
\begin{aligned}
\left\langle\tau, \tilde{\mathrm{q}}_{\mathrm{c}}(\mathrm{x})\right\rangle= & \left\langle\sigma, \mathrm{Q}_{1}(\mathrm{x})\left(\phi_{\mathrm{c}}(\mathrm{x})-\psi(\mathrm{x})\right)\right\rangle+\left\langle\sigma, \mathrm{r}_{1} \mathrm{q}_{\mathrm{c}}(\mathrm{x})\right\rangle \\
& -\left\langle\sigma, \mathrm{Q}_{2}(\mathrm{x}) \phi(x)\right\rangle-\left\langle\sigma, \mathrm{r}_{2} \phi_{\mathrm{c}}(\mathrm{x})\right\rangle \\
= & \left\langle\sigma, \mathrm{Q}_{3}(\mathrm{x}) \phi(\mathrm{x})\right\rangle+\left\langle\sigma, \mathrm{r}_{3} \phi_{\mathrm{c}}(\mathrm{x})\right\rangle \\
& -\left\langle\sigma, \mathrm{Q}_{1}(\mathrm{x}) \psi(\mathrm{x})\right\rangle+\left\langle\sigma, \mathrm{r}_{1} \mathrm{q}_{\mathrm{c}}(\mathrm{x})\right\rangle \\
& -\left\langle\sigma, \mathrm{Q}_{2}(\mathrm{x}) \phi(x)\right\rangle-\left\langle\sigma, \mathrm{r}_{2} \phi_{\mathrm{c}}(\mathrm{x})\right\rangle \\
= & \left\langle\phi(x) \sigma, \mathrm{Q}_{3}(\mathrm{x})\right\rangle+\left\langle\phi(\mathrm{x}) \sigma, \mathrm{Q}_{1}^{\prime}(\mathrm{x})\right\rangle \\
& -\left\langle\phi(x) \sigma, \mathrm{Q}_{2}(x)\right\rangle+\mathrm{r}_{1}\left\langle\sigma, \mathrm{q}_{\mathrm{c}}(\mathrm{x})\right\rangle \\
= & \mathrm{r}_{1}\left\langle\sigma, \mathrm{q}_{\mathrm{c}}(\mathrm{x})\right\rangle=\prod_{\mathrm{l}=1}^{\mathrm{m}}\left(\mathrm{c}-\mathrm{c}_{\mathrm{l}}\right)^{\mathrm{m}_{\mathrm{l}}+2}\left\langle\sigma, \mathrm{q}_{\mathrm{c}}(\mathrm{x})\right\rangle \neq 0 .
\end{aligned}
$$

Hence $\mathbb{N}(\tau ; c) \neq 0$.

\section{Examples}

As illustrating examples, we consider the following example.

Example 4.1. Let

$$
\tau:=\sigma+\lambda\left(u_{10} \delta(x-1)+u_{20} \delta(x+1)+u_{11} \delta^{\prime}(x-1)+u_{21} \delta^{\prime}(x+1)\right),
$$


where $\lambda \neq 0,\left|u_{10}\right|+\left|u_{20}\right|+\left|u_{11}\right|+\left|u_{21}\right| \neq 0$, and $\sigma$ is the Jacobi moment functional defined by

$$
\langle\sigma, \pi\rangle=\int_{-1}^{1}(1-x)^{\alpha}(1+x)^{\beta} \pi(x) d x \quad(\alpha>-1, \beta>-1), \quad \pi \in \mathbb{P} .
$$

Then

$$
\begin{aligned}
P_{n}(x) & =P_{n}^{(\alpha, \beta)}(x) \\
& =\left(\begin{array}{c}
2 n+\alpha+\beta \\
n
\end{array}\right)^{-1} \sum_{k=0}^{n}\left(\begin{array}{c}
n+\alpha \\
n-k
\end{array}\right)\left(\begin{array}{c}
n+\beta \\
k
\end{array}\right)(x-1)^{k}(x+1)^{n-k}, \quad n \geq 0
\end{aligned}
$$

are the Jacobi polynomials satisfying

$$
\begin{aligned}
\left(1-x^{2}\right) y^{\prime \prime}(x)+ & {[\beta-\alpha-(\alpha+\beta+2) x] y^{\prime}(x)+n(n+\alpha+\beta+1) y(x)=0, } \\
\left\langle\sigma, P_{n}^{(\alpha, \beta)}(x)^{2}\right\rangle & :=k_{n} \\
& =\frac{2^{\alpha+\beta+2 n+1} n ! \Gamma(n+\alpha+1) \Gamma(n+\beta+1)}{\Gamma(n+\alpha+\beta+1)(2 n+\alpha+\beta+1)(n+\alpha+\beta+1)_{n}^{2}}, \quad n \geq 0,
\end{aligned}
$$

where

$$
(a)_{k}=\left\{\begin{array}{l}
1, \quad \text { if } k=0 \\
a(a+1) \cdots(a+k-1), \quad \text { if } k \geq 1 .
\end{array}\right.
$$

In this case, using the differential-difference relation

$$
\left(P_{n}^{(\alpha, \beta)}(x)\right)^{(v)}=\frac{n !}{(n-v) !} P_{n-v}^{(\alpha+v, \beta+v)}(x), \quad v=0,1,2, \ldots, n \geq v
$$

the structure relation

$$
\left(1-x^{2}\right) P_{n}^{(\alpha, \beta)}(x)^{\prime}=\tilde{\alpha}_{n} P_{n+1}^{(\alpha, \beta)}(x)+\tilde{\beta}_{n} P_{n}^{(\alpha, \beta)}(x)+\tilde{\gamma}_{n} P_{n-1}^{(\alpha, \beta)}(x), \quad n \geq 0,
$$

where

$$
\begin{aligned}
& \tilde{\alpha}_{n}=-n \\
& \tilde{\beta}_{n}=\frac{2(\alpha-\beta) n(n+\alpha+\beta+1)}{(2 n+\alpha+\beta)(2 n+2+\alpha+\beta)} \\
& \tilde{\gamma}_{n}=\frac{4 n(n+\alpha)(n+\beta)(n+\alpha+\beta)(n+\alpha+\beta+1)}{(2 n+\alpha+\beta-1)(2 n+\alpha+\beta)^{2}(2 n+\alpha+\beta+1)},
\end{aligned}
$$

and the three term recurrence relation

$$
P_{n+1}^{(\alpha, \beta)}(x)=\left(x-\beta_{n}\right) P_{n}^{(\alpha, \beta)}(x)-\gamma_{n} P_{n-1}^{(\alpha, \beta)}(x),
$$




\section{Generalized Uvarov transforms}

where

$$
\begin{aligned}
& \beta_{n}=\frac{\beta^{2}-\alpha^{2}}{(2 n+\alpha+\beta)(2 n+2+\alpha+\beta)} \\
& \gamma_{n}=\frac{4 n(n+\alpha)(n+\beta)(n+\alpha+\beta)}{(2 n+\alpha+\beta-1)(2 n+\alpha+\beta)^{2}(2 n+\alpha+\beta+1)},
\end{aligned}
$$

we can easily obtain (see [1, equations (30)-(32)]):

$$
\begin{aligned}
K_{n-1}^{(0,0)}(1,1)= & \frac{\left(P_{n}^{(\alpha, \beta)}(1)\right)^{2} n(n+\beta)}{k_{n-1}(2 n+\alpha+\beta+1) \gamma_{n}(\alpha+1)}, \\
K_{n-1}^{(0,0)}(1,-1)= & -\frac{n P_{n}^{(\alpha, \beta)}(-1) P_{n}^{(\alpha, \beta)}(1)}{k_{n-1}(2 n+\alpha+\beta+1) \gamma_{n}}, \\
K_{n-1}^{(0,1)}(1,1)= & \frac{\left(P_{n}^{(\alpha, \beta)}\right)^{\prime}(1) P_{n}^{(\alpha, \beta)}(1)(n+\beta)(n-1)}{k_{n-1}(2 n+\alpha+\beta+1) \gamma_{n}(\alpha+2)}, \\
K_{n-1}^{(0,1)}(1,-1)= & -\frac{\left(P_{n}^{(\alpha, \beta)}\right)^{\prime}(-1) P_{n}^{(\alpha, \beta)}(1)(n-1)}{k_{n-1}(2 n+\alpha+\beta+1) \gamma_{n}}, \\
K_{n-1}^{(1,1)}(1,1)= & P_{n}^{(\alpha, \beta)}(1)\left(P_{n}^{(\alpha, \beta)}\right)^{\prime}(1)(n-1)(n+\beta) \\
& \times \frac{\left[(\alpha+2)\left(n^{2}+n \alpha+n \beta\right)-(\alpha+1)(\alpha+\beta+2)\right]}{2 k_{n-1}(2 n+\alpha+\beta+1) \gamma_{n}(\alpha+1)(\alpha+2)(\alpha+3)}, \\
K_{n-1}^{(0,0)}(1,1)= & -\frac{P_{n}^{(\alpha, \beta)}(1)\left(P_{n}^{(\alpha, \beta)}\right)^{\prime}(-1)(n-1)\left[n^{2}+n \alpha+n \beta-\alpha-\beta-2\right]}{2 k_{n-1}(2 n+\alpha+\beta+1) \gamma_{n}(\alpha+1)},
\end{aligned}
$$

where

$$
K_{n}(x, y)=\sum_{k=0}^{n} \frac{P_{k}^{(\alpha, \beta)}(x) P_{k}^{(\alpha, \beta)}(y)}{k_{n}}
$$

is the $n$th kernel polynomial of $\left\{P_{n}^{(\alpha, \beta)}(x)\right\}_{n=0}^{\infty}$ and $K_{n}^{(i, j)}(x, y)=\partial_{x}^{i} \partial_{y}^{j} K_{n}(x, y)$. Using the symmetry of the Jacobi kernels, we obtain that the moment functional $\tau$ in (4.1) is quasi-definite if and only if

$$
d_{n}=\left|\begin{array}{ll}
A_{11} & A_{12} \\
A_{21} & A_{22}
\end{array}\right| \neq 0, \quad n \geq 0,
$$


where

$$
\begin{aligned}
& A_{11}=\left(\begin{array}{cc}
1+\lambda u_{10} K_{n}^{(0,0)}(1,1)+\lambda u_{11} K_{n}^{(0,1)}(1,1) & \lambda u_{11} K_{n}^{(0,0)}(1,1) \\
\lambda u_{10} K_{n}^{(1,0)}(1,1)+\lambda u_{11} K_{n}^{(1,1)}(1,1) & 1+\lambda u_{11} K_{n}^{(1,0)}(1,1)
\end{array}\right) \\
& A_{12}=\left(\begin{array}{cc}
\lambda u_{20} K_{n}^{(0,0)}(1,-1)+\lambda u_{21} K_{n}^{(0,1)}(1,-1) & \lambda u_{21} K_{n}^{(0,0)}(1,-1) \\
\lambda u_{20} K_{n}^{(1,0)}(1,-1)+\lambda u_{21} K_{n}^{(1,1)}(1,-1) & \lambda u_{21} K_{n}^{(1,0)}(1,-1)
\end{array}\right) \\
& A_{21}=\left(\begin{array}{lr}
\lambda u_{10} K_{n}^{(0,0)}(-1,1)+\lambda u_{11} K_{n}^{(0,1)}(-1,1) & \lambda u_{11} K_{n}^{(0,0)}(-1,1) \\
\lambda u_{10} K_{n}^{(1,0)}(-1,1)+\lambda u_{11} K_{n}^{(1,1)}(-1,1) & \lambda u_{11} K_{n}^{(1,0)}(-1,1)
\end{array}\right) \\
& A_{22}= \\
& \left(\begin{array}{cc}
1+\lambda u_{20} K_{n}^{(0,0)}(-1,-1)+\lambda u_{21} K_{n}^{(0,1)}(-1,-1) & \lambda u_{21} K_{n}^{(0,0)}(-1,-1) \\
\lambda u_{20} K_{n}^{(1,0)}(-1,-1)+\lambda u_{21} K_{n}^{(1,1)}(-1,-1) & 1+\lambda u_{21} K_{n}^{(1,0)}(-1,-1)
\end{array}\right) .
\end{aligned}
$$

Álvarez-Nodarse, J. Arvesú, and F. Marcellán [1] showed that for any values of $\lambda$ and $u_{10}, u_{20}, u_{11}, u_{21}, d_{n} \neq 0$ for large $n$ so that $R_{n}(x)$ exists for large $n$. Moreover, they express $R_{n}(x)$ as

$$
\begin{aligned}
R_{n}(x)= & \left(1+n \zeta_{n}+n \eta_{n}\right) P_{n}^{(\alpha, \beta)}(x)+\left[\zeta_{n}(1-x)-\eta_{n}(1+x)+\theta_{n}\right]\left(P_{n}^{(\alpha, \beta)}(x)\right)^{\prime} \\
& +\left[\chi_{n}(1+x)-\omega_{n}(1-x)\right]\left(P_{n}^{(\alpha, \beta)}(x)\right)^{\prime \prime}
\end{aligned}
$$

where $\zeta_{n}, \eta_{n}, \theta_{n}, \chi_{n}$, and $\omega_{n}$ are constants depending on $n, \lambda, u_{10}, u_{20}$, and $u_{11}$, (see [1, equations (47)-(50)]). They also express $R_{n}(x)$ as a generalized hypergeometric series ${ }_{6} F_{5}$ (see [1, Proposition 2]).

Example 4.2. Consider a moment functional $\tau$ given by

$$
\tau:=\sigma+\lambda \sum_{k=0}^{N} \frac{(-1)^{k} u_{k}}{k !} \delta^{(k)}(x),
$$

where $\lambda \neq 0, u_{k} \in \mathbb{C}, N \in\{0,1,2, \ldots\}$ and $\sigma$ is the Laguerre moment functional defined by

$$
\langle\sigma, p\rangle=\int_{0}^{\infty} x^{\alpha} e^{-x} \pi(x) d x \quad(\alpha>-1), \pi \in \mathbb{P} .
$$

Then

$$
\begin{aligned}
P_{n}(x) & =L_{n}^{(\alpha)}(x)=(-1)^{n} n ! \sum_{k=0}^{n}\left(\begin{array}{l}
n+\alpha \\
n-k
\end{array}\right) \frac{(-x)^{k}}{k !} \\
& =(-1)^{n}(\alpha+1)_{n}{ }_{1} F_{1}(-n ; \alpha+1 ; x), \quad n \geq 0
\end{aligned}
$$


are the monic Laguerre polynomials satisfying

$$
\begin{aligned}
& x y^{\prime \prime}(x)+(1+\alpha-x) y^{\prime}(x)+n y(x)=0 \\
& \left\langle\sigma, L_{n}^{(\alpha)}(x)^{2}\right\rangle=n ! \Gamma(n+\alpha+1), \quad n \geq 0 .
\end{aligned}
$$

Hence

$$
\mathrm{L}_{n}^{(\alpha)}(0)=\frac{(-1)^{n} \Gamma(n+\alpha+1)}{\Gamma(\alpha+1)}=(-1)^{n}\left(\begin{array}{c}
n+\alpha \\
n
\end{array}\right) n !
$$

Hence, by Theorem 2.2, the moment functional $\tau$ in (4.16) is quasi-definite if and only if $d_{n} \neq 0$, where $d_{n}$ is the determinant of the $(N+1) \times(N+1)$ matrix $D_{n}$,

$$
D_{n}:=\left[\delta_{i j}+\lambda \sum_{k=0}^{N-j} \frac{u_{j+k}}{j ! k !} K_{n}^{(i, k)}(0,0)\right]_{i, j=0}^{N}, \quad n \geq 0
$$

where $K_{n}(x, y)=\sum_{k=0}^{n} L_{k}^{(\alpha)}(x) L_{k}^{(\alpha)}(y) /\left\langle\sigma, L_{k}^{(\alpha)}(x)^{2}\right\rangle$.

When $d_{n} \neq 0$ for $n \geq 0$, we now claim that the MOPS $\left\{R_{n}(x)\right\}_{n=0}^{\infty}$ relative to $\tau$ can be given as

$$
R_{n}(x)=\sum_{k=0}^{N+1} A_{k}^{(n)} \partial_{x}^{k} L_{n}^{(\alpha)}(x), \quad n \geq 0
$$

for suitable constants $A_{k}^{(n)}(0 \leq k \leq N+1)$ with $A_{0}^{(n)}=1$. For any fixed $n \geq 1$, set

$$
\tilde{R}_{n}(x):=\sum_{k=0}^{N+1} A_{k} \partial_{x}^{k} L_{n}^{(\alpha)}(x)
$$

where $\left\{A_{k}\right\}_{k=0}^{N+1}$ are constants to be determined. Note here that if $0 \leq n \leq N$, then $\partial_{x}^{k} L_{n}^{(\alpha)}(x)=0$ for $n+1 \leq k \leq N+1$ so that we may take $A_{k}$ for $n+1 \leq k \leq N+1$ to be 0 . Since $\left(L_{n}^{(\alpha)}(x)\right)^{\prime}=n L_{n-1}^{(\alpha+1)}(x), n \geq 1$, we have

$$
\tilde{R}_{n}(x)=\sum_{k=0}^{N+1}(n-k+1)_{k} A_{k} L_{n-k}^{(\alpha+k)}(x)
$$

where $L_{n}^{(\alpha)}(x)=0$ for $n<0$. We now show that the coefficients $\left\{A_{k}\right\}_{k=0}^{N+1}$ can 
be chosen so that

$$
\left\langle\tau, x^{m} \tilde{R}_{n}(x)\right\rangle=0, \quad 0 \leq m \leq n-1
$$

If $\mathrm{N}+1 \leq \mathrm{m}<\mathrm{n}$, then by (4.24)

$$
\begin{aligned}
\left\langle\tau, x^{m} \tilde{R}_{n}(x)\right\rangle & =\int_{0}^{\infty} x^{m} \tilde{R}_{n}(x) x^{\alpha} e^{-x} d x+\lambda \sum_{k=0}^{N} \frac{u_{k}}{k !}\left(x^{m} \tilde{R}_{n}(x)\right)^{(k)}(0) \\
& =\sum_{k=0}^{N+1} \frac{n !}{(n-k) !} A_{k} \int_{0}^{\infty} x^{m} L_{n-k}^{(\alpha+k)}(x) x^{\alpha} e^{-x} d x \\
& =\sum_{k=0}^{N+1} \frac{n !}{(n-k) !} A_{k} \int_{0}^{\infty} x^{m-k} L_{n-k}^{(\alpha+k)}(x) x^{\alpha+k} e^{-x} d x \\
& =0
\end{aligned}
$$

We now assume that $0 \leq m \leq \min (N, n-1)$. Then

$$
\begin{aligned}
\left\langle\sigma, x^{m} L_{n-k}^{(\alpha+k)}(x)\right\rangle & =\int_{0}^{\infty} x^{m} L_{n-k}^{(\alpha+k)}(x) x^{\alpha} e^{-x} d x \\
& =\int_{0}^{\infty} x^{m-k} L_{n-k}^{(\alpha+k)}(x) x^{\alpha+k} e^{-x} d x=0
\end{aligned}
$$

for $0 \leq k \leq m$. For $m+1 \leq k \leq n$,

$$
\begin{aligned}
\left\langle\sigma, x^{m} L_{n-k}^{(\alpha+k)}(x)\right\rangle= & (-1)^{n-k}(n-k) ! \sum_{j=0}^{n-k} \frac{(-1)^{j}}{j !}\left(\begin{array}{c}
n+\alpha \\
n-k-j
\end{array}\right) \int_{0}^{\infty} x^{m+\alpha+j} e^{-x} d x \\
= & (-1)^{n-k}(n-k) ! \sum_{j=0}^{n-k} \frac{(-1)^{j}}{j !}\left(\begin{array}{c}
n+\alpha \\
n-k-j
\end{array}\right) \Gamma(m+\alpha+j+1) \\
= & (-1)^{n-k}(n-k) !\left(\begin{array}{c}
n+\alpha \\
n-k
\end{array}\right) \Gamma(m+\alpha+1) \\
& \times{ }_{2} F_{1}(-n+k, m+\alpha+1 ; k+\alpha+1 ; 1) \\
= & (-1)^{n-k}(n-k) !\left(\begin{array}{c}
n-m-1 \\
n-k
\end{array}\right) \Gamma(m+\alpha+1)
\end{aligned}
$$


by $(4.18)$ and ${ }_{2} F_{1}(-n, b ; c ; 1)=(c-b)_{n} /(c)_{n}$. Hence by $(4.20)$

$$
\begin{aligned}
&\left\langle\tau, x^{m} \tilde{R}_{n}(x)\right\rangle= \sum_{k=0}^{N+1}(n-k+1)_{k} A_{k}\left\langle\sigma, x^{m} L_{n-k}^{(\alpha+k)}(x)\right\rangle \\
&+\lambda \sum_{l=0}^{N} \frac{u_{l}}{l !} \sum_{k=0}^{N+1}(n-k+1)_{k} A_{k}\left(x^{m} L_{n-k}^{(\alpha+k)}(x)\right)^{(l)}(0) \\
&= n ! \Gamma(m+\alpha+1) \sum_{k=m+1}^{N+1}(-1)^{n-k}\left(\begin{array}{c}
n-m-1 \\
n-k
\end{array}\right) A_{k} \\
&+\lambda n ! \sum_{l=m}^{N} \frac{u_{l}}{(l-m) !} \sum_{k=0}^{N+1}(-1)^{n-k-l+m}\left(\begin{array}{c}
n+\alpha \\
n-k-l+m
\end{array}\right) A_{k}, \\
& 0 \leq m \leq m i n(N, n-1),
\end{aligned}
$$

where $\left(\begin{array}{l}n \\ k\end{array}\right)=0$, for $k<0$. Hence $\left\langle\tau, x^{m} \tilde{R}_{n}(x)\right\rangle=0$, with $0 \leq m \leq n-1$ if and only if

$$
\begin{aligned}
& \Gamma(m+\alpha+1) \sum_{k=m+1}^{N+1}(-1)^{n-k}\left(\begin{array}{c}
n-m-1 \\
n-k
\end{array}\right) A_{k} \\
& +\lambda \sum_{l=m}^{N} \frac{u_{l}}{(l-m) !} \sum_{k=0}^{N+1}(-1)^{n-k-l+m}\left(\begin{array}{c}
n+\alpha \\
n-k-l+m
\end{array}\right) A_{k}=0 \\
& \quad 0 \leq m \leq \min (N, n-1) .
\end{aligned}
$$

Since (4.30) is a homogeneous system of $N+1$ (resp., $n$ ) equations for $N+2$ (resp., $n+1$ ) unknowns $\left\{A_{k}\right\}_{k=0}^{N+1}$ (resp., $\left\{A_{k}\right\}_{k=0}^{n}$ ) when $n \geq N+1$ (resp., $n \leq N)$, there always exists a nontrivial solution $\left\{A_{k}\right\}_{k=0}^{N+1}$. With this choice of $\left\{A_{k}\right\}_{k=0}^{N+1}, \tilde{R}_{n}(x)$ is a nonzero polynomial of degree $\leq n$ and $\left\langle\tau, x^{m} \tilde{R}_{n}(x)\right\rangle=0$ for $0 \leq m \leq n-1$ so that $\operatorname{deg}\left(\tilde{R}_{n}\right)=n$, that is, $A_{0} \neq 0$. Then $A_{0}^{-1} \tilde{R}_{n}(x)=R_{n}(x)$ so that we have (4.22).

Now we can express $R_{n}(x)$ as a hypergeometric series (see [13]);

$$
\begin{aligned}
R_{n}(x)= & \frac{\beta_{0} \beta_{1} \cdots \beta_{N}}{(\alpha+1)_{N+1}}(-1)^{n}(\alpha+1)_{n}\left(A_{0}+A_{1}+\cdots+A_{N+1}\right) \\
& \times{ }_{N+2} F_{N+2}\left(\begin{array}{c}
-n, \beta_{0}+1, \beta_{1}+1, \ldots, \beta_{N}+1 \\
\alpha+N+2, \beta_{0}, \beta_{1}, \ldots, \beta_{N}
\end{array} \mid x\right)
\end{aligned}
$$

for suitable constants $\left\{\beta_{j}\right\}_{j=0}^{N}$. 


\section{Acknowledgements}

D. H. Kim was partially supported by BK-21 project and K. H. Kwon was partially supported by KOSEF (99-2-101-001-5).

\section{References}

[1] R. Álvarez-Nodarse, J. Arvesú, and F. Marcellán, A generalization of the JacobiKoornwinder polynomials, preprint.

[2] R. Álvarez-Nodarse and F. Marcellán, A generalization of the class of Laguerre polynomials: asymptotic properties and zeros, Appl. Anal. 62 (1996), no. 34, 349-366. Zbl 866.33005.

[3] J. Arvesú, R. Álvarez-Nodarse, F. Marcellán, and K. H. Kwon, Some extension of the Bessel-type orthogonal polynomials, Integral Transform. Spec. Funct. 7 (1998), no. 3-4, 191-214. MR 2001b:33013. Zbl 936.33003.

[4] S. Belmehdi and F. Marcellán, Orthogonal polynomials associated with some modifications of a linear functional, Appl. Anal. 46 (1992), no. 1-2, 1-24. MR 94f:33012. Zbl 807.39013.

[5] C. Bernardi and Y. Maday, Some spectral approximations of one-dimensional fourth-order problems, Progress in Approximation Theory, Academic Press, Massachusetts, 1991, pp. 43-116. MR 92j:65176.

[6] S. Bochner, Über Sturm-Liouvillesche polynomsysteme, Math. Z. 29 (1929), 730-736.

[7] T. S. Chihara, An Introduction to Orthogonal Polynomials, Mathematics and its Applications, vol. 13, Gordon and Breach Science Publishers, New York, 1978. MR 58\#1979. Zbl 389.33008.

[8] - Orthogonal polynomials and measures with end point masses, Rocky Mountain J. Math. 15 (1985), no. 3, 705-719. MR 87a:42037. Zbl 586.33007.

[9] N. Draïdi and P. Maroni, Sur l'adjonction de deux masses de Dirac à une forme régulière quelconque [On the adjointness of two Dirac masses to an arbitrary regular form ], Orthogonal Polynomials and their Applications (Spanish) (Vigo, 1988), Esc. Téc. Super. Ing. Ind. Vigo, Vigo, 1989, pp. 83-90 (French). MR 92m:42024. Zbl 764.42015.

[10] A. A. Gonchar, On convergence of Pade approximants for some classes of meromorphic functions, Math. USSR, Sb. 26 (1975), 555-575, [translated from Mat. Sb. 97 (1975) 607-629]. Zbl 341.30029.

[11] E. Hendriksen, A Bessel type orthogonal polynomial system, Nederl. Akad. Wetensch. Indag. Math. 46 (1984), no. 4, 407-414. MR 86c:33016. Zbl 564.33006 .

[12] D. H. Kim, K. H. Kwon, and S. B. Park, Delta perturbation of a moment functional, Appl. Anal. 74 (2000), no. 3-4, 463-477. MR 2001c:33018.

[13] R. Koekoek, Generalizations of Laguerre polynomials, J. Math. Anal. Appl. 153 (1990), no. 2, 576-590. MR 92d:33016.

[14] T. H. Koornwinder, Orthogonal polynomials with weight function $(1-x)^{\alpha}(1+$ $x)^{\beta}+M \delta(x+1)+N \delta(x-1)$, Canad. Math. Bull. 27 (1984), no. 2, 205-214. MR 85i:33011. Zbl 532.33010.

[15] A. M. Krall, Orthogonal polynomials satisfying fourth order differential equations, Proc. Roy. Soc. Edinburgh Sect. A 87 (1980/81), no. 3-4, 271-288. MR 82d:33021. Zbl 453.33006. 
[16] H. L. Krall, On orthogonal polynomials satisfying a certain fourth order differential equation, Pennsylvania State College Studies, 1940 (1940), no. 6, 24. MR 2,98a. Zbl 060.19210.

[17] K. H. Kwon and J. H. Lee, Division problem of moment functionals, submitted.

[18] K. H. Kwon and L. L. Littlejohn, Classification of classical orthogonal polynomials, J. Korean Math. Soc. 34 (1997), no. 4, 973-1008. MR 99k:33028. Zbl 898.33002.

[19] K. H. Kwon, L. L. Littlejohn, and G. J. Yoon, Orthogonal polynomial solutions to spectral type differential equations; Magnus' Conjecture, submitted.

[20] K. H. Kwon and S. B. Park, Two-point masses perturbation of regular moment functionals, Indag. Math. (N.S.) 8 (1997), no. 1, 79-93. MR 99d:42044. Zbl 877.42009.

[21] G. L. Lopes, Convergence of Pade approximants of Stieltjes type meromorphic functions and comparative asymptotics for orthogonal polynomials, Math. USSR, Sb. 64 (1989), 207-227, [translated from Mat. Sb. 136 (1988) 206226]. Zbl 669.30026.

[22] A. P. Magnus, Associated Askey-Wilson polynomials as Laguerre-Hahn orthogonal polynomials, Orthogonal Polynomials and their Applications (Segovia, 1986), Lecture Notes in Math., vol. 1329, Springer, Berlin, 1988, pp. 261-278. MR 90d:33008. Zbl 645.33015.

[23] F. Marcellán and P. Maroni, Sur l'adjonction d'une masse de Dirac à une forme régulière et semi-classique [On the assignment of a Dirac-mass for a regular and semi-classical form], Ann. Mat. Pura Appl. (4) 162 (1992), 1-22 (French). MR 94e:33014. Zbl 771.33008.

[24] P. Maroni, Prolégomènes à l'étude des polynômes orthogonaux semi-classiques [Preliminary remarks for the study of semi-classical orthogonal polynomials], Ann. Mat. Pura Appl. (4) 149 (1987), 165-184 (French). MR 89c:33016. Zbl 636.33009.

[25] - Variations around classical orthogonal polynomials. Connected problems, J. Comput. Appl. Math. 48 (1993), no. 1-2, 133-155. MR 94k:33013. Zbl 790.33006.

[26] An introduction to second degree forms, Adv. Comput. Math. 3 (1995), no. 1-2, 59-88. MR 96c:42053. Zbl 837.42009.

[27] P. G. Nevai, Orthogonal polynomials, Mem. Amer. Math. Soc. 18 (1979), no. 213, 1-185. MR 80k:42025. Zbl 405.33009.

[28] V. B. Uvarov, The connection between systems of polynomials orthogonal with respect to different distribution functions, U.S.S.R. Comput. Math. and Math. Phys. 9 (1969), 25-36. Zbl 231.42013.

[29] A. Zhedanov, Rational spectral transformations and orthogonal polynomials, J. Comput. Appl. Math. 85 (1997), no. 1, 67-86. MR 98h:42026. Zbl 918.42016.

D. H. Kim: Division of Applied Mathematics, Korea Advanced Institute of Science and Technology, Taejon 305-701, Korea

E-mail address:dhkim@jacobi.kaist.ac.kr

K. H. Kwon: Division of Applied Mathematics, Korea Advanced Institute of Science and Technology, Taejon 305-701, Korea

E-mail address: khkwon@jacobi.kaist.ac.kr 


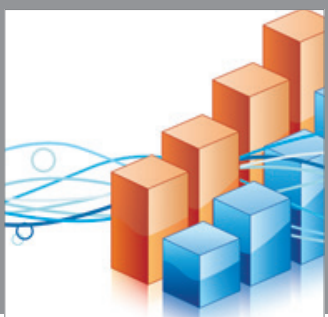

Advances in

Operations Research

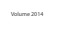

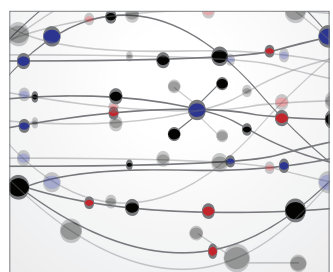

\section{The Scientific} World Journal
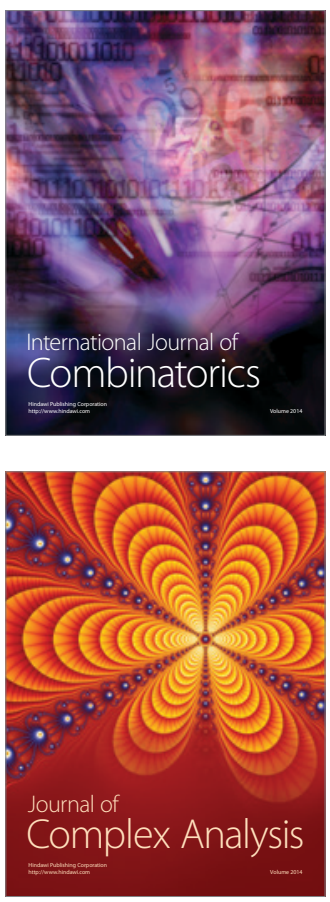

International Journal of

Mathematics and

Mathematical

Sciences
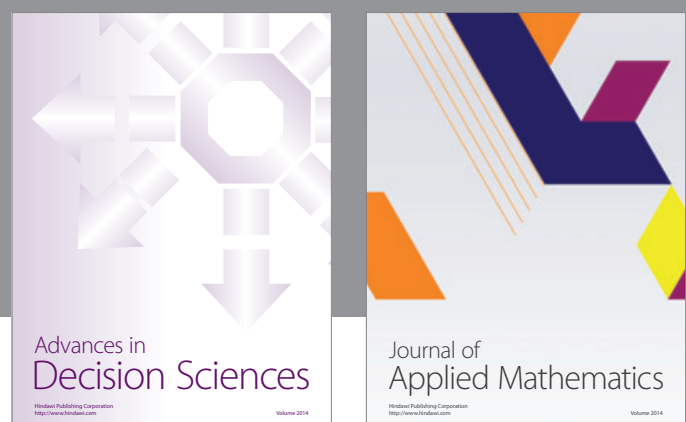

Journal of

Applied Mathematics
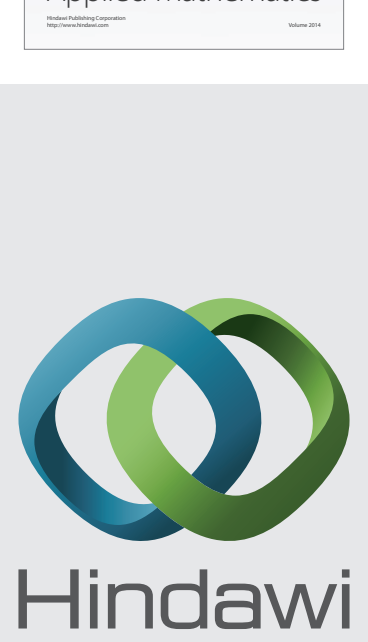

Submit your manuscripts at http://www.hindawi.com
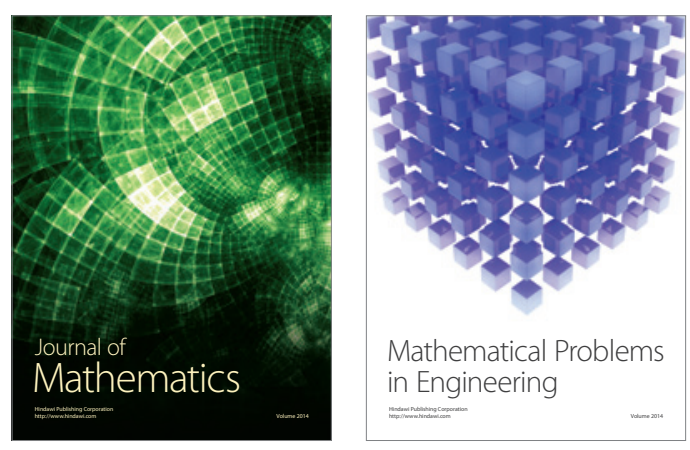

Mathematical Problems in Engineering
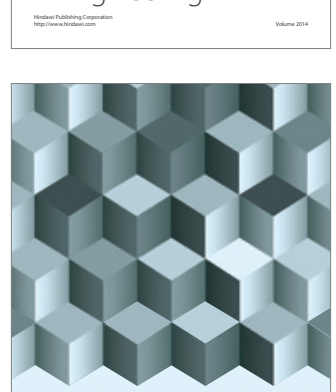

Journal of

Function Spaces
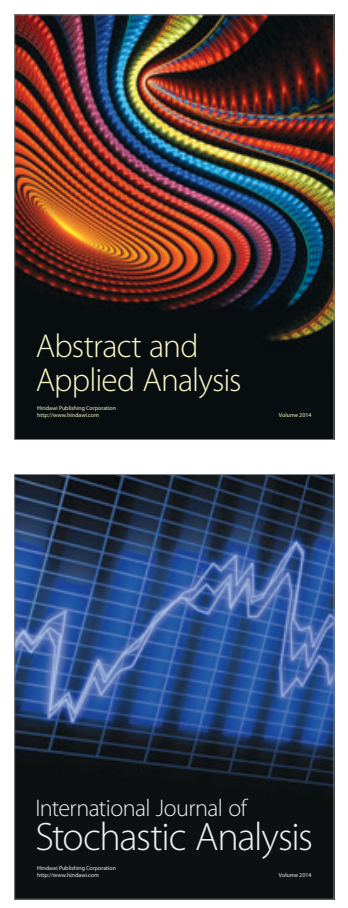

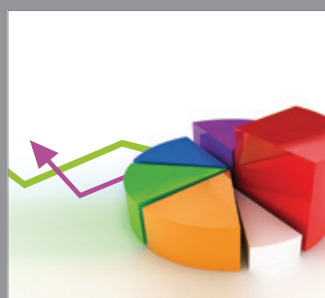

ournal of

Probability and Statistics

Promensencen
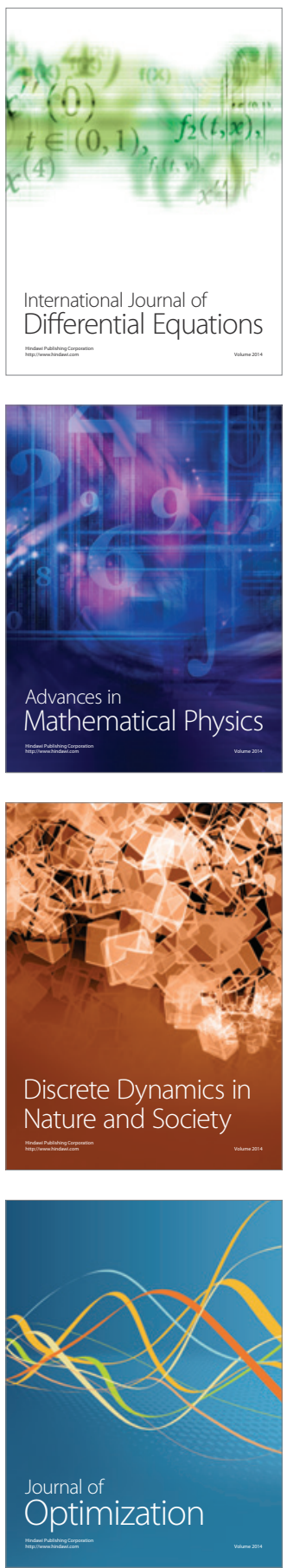